\title{
Forethought in Youth with Attention Deficit/Hyperactivity Disorder: An fMRI Study of Sex-Specific Differences
}

\author{
H. Poissant, ${ }^{1}$ L. Rapin, ${ }^{2}$ S. Chenail, ${ }^{3}$ and A. Mendrek ${ }^{3}$ \\ ${ }^{1}$ Department of Education and Pedagogy, UQAM, Montreal, QC, Canada H2L 2C4 \\ ${ }^{2}$ Department of Linguistics, UQAM, Montreal, QC, Canada H2L 2C4 \\ ${ }^{3}$ Department of Psychology, Bishop's University, Sherbrooke, QC, Canada J1M $1 Z 7$
}

Correspondence should be addressed to H. Poissant; poissant.helene@uqam.ca

Received 21 December 2015; Accepted 3 May 2016

Academic Editor: Umberto Albert

Copyright (C) 2016 H. Poissant et al. This is an open access article distributed under the Creative Commons Attribution License, which permits unrestricted use, distribution, and reproduction in any medium, provided the original work is properly cited.

\begin{abstract}
Objective. The majority of studies investigating neurocognitive processing in attention deficit/hyperactivity disorder (ADHD) have been conducted on male participants. Few studies evaluated females or examined sex differences. Among various cognitive anomalies in ADHD, deficit in forethought seems particularly important as children with ADHD often fail to adequately use previous information in order to prepare for responses. The main goal of this study was to assess sex-specific differences in behavioral and neural correlates of forethought in youth with ADHD. Methods. 21 typically developing (TD) youth and 23 youth with ADHD were asked to judge whether two pictures told a congruent or incongruent story. Reaction time, performance accuracy, and cerebral activations were recorded during functional magnetic resonance imaging (fMRI). Results. Significant sex-specific differences in cerebral activations appeared, despite equivalent performance. Relative to the boys TD participants, boys with ADHD had extensive bilateral frontal and parietal hypoactivations, while girls with ADHD demonstrated more scattered hypoactivations in the right cerebral regions. Conclusion. Present results revealed that youth with ADHD exhibit reduced cerebral activations during forethought. Nevertheless, the pattern of deficits differed between boys and girls, suggesting the use of a different neurocognitive strategy. This emphasizes the importance of including both genders in the investigations of ADHD.
\end{abstract}

\section{Introduction}

Attention deficit/hyperactive disorder (ADHD) is a neurodevelopmental disorder defined by persistent inattention and/or hyperactivity/impulsivity [1]. The disorder negatively impacts social, academic, or occupational functioning. Most of the studies have been conducted on male participants resulting in a limited knowledge regarding sex and gender differences in ADHD [2]. The higher prevalence of ADHD in boys could partly explain this gap in the literature [3], but we need more information regarding ADHD females, especially in the realm of neurocognitive dysfunction. Thus, the present study was designed to examine neural sex differences in ADHD.

The expression of ADHD appears to be sexually dimorphic. Boys seem to exhibit more hyperactivity, inattention, impulsivity, and externalizing problems. Girls generally show more internalizing problems (depression, anxiety) and intellectual impairment $[4,5]$. Larger deficits in inhibition have also been reported in males [6]. Differences in age of onset and differences in symptoms could be due to neurochemical and hormonal factors. For example, boys show a significant increase in dopamine (DA) receptor density before and during puberty, which could contribute to more hyperactivity symptoms at an earlier stage [7]. In comparison, girls demonstrate a later increase in DA receptors related to an increase in estrogen during puberty [7].

Several executive dysfunctions have been found in children with ADHD, including difficulties with prioritizing, time management, and initiating and completing tasks as well as difficulty in shifting cognitive sets and poor working memory (WM) [8]. Working memory, defined as the ability to retain information for prospective execution of an action [9], seems specifically affected in ADHD [3]. A meta-analysis indicated that children with ADHD scored lower than control children on verbal storage, verbal central executive, visualspatial storage, and visual-spatial central executive [10]. 
Another meta-analysis [11] found significant patterns of hypoactivity in ADHD, which affected the anterior cingulate cortex (ACC), dorsolateral and inferior prefrontal cortex (PFC), basal ganglia, thalamus, and portions of the parietal cortex. Other findings also point to deficits in the neural activity of the frontostriatal and frontoparietal pathways [12]. Studies on inhibition alone indicate hypoactivation, especially pronounced in the PFC, and greater activity in the medial frontal gyrus and the parietal paracentral lobule in individuals with ADHD [11].

Only a few neuroimaging studies on ADHD have included females. The percentage of females across all studies has been estimated to be less than $20 \%$ [2] while the male to female ratio among ADHD is estimated to be $2.28: 1$ [13]. Valera and colleagues (2010) [14] found that while performing a verbal WM task, males with ADHD showed significantly less activation in the right frontal, right temporal, left occipital, cerebellar, and some subcortical regions. However, no differences in activation were found between females with and without ADHD. Similar results were obtained in a subsequent fMRI study [15], while the electroencephalography (EEG) investigation revealed markedly different EEG profiles in boys and girls with ADHD [16].

Forethought is a prospective function of WM related to the ability to conjecture possible future related events [17]. Children with ADHD often fail to adequately use previous information in order to prepare for upcoming responses. One study found that children with ADHD showed diminished activation in the ventral PFC and ACC when having to react to a stimulus that was not expected from a past stimulus [18]. More recently, Poissant et al. (2012) [19] also found hypoactivation of the PFC in youth with ADHD and higher reaction times during a task requiring forethought.

In the present study we examined neural sex differences in ADHD during a forethought task. Based on previous findings, it was expected that boys with ADHD would demonstrate poorer performance and greater cerebral hypoactivation during the task relative to girls with ADHD.

\section{Method}

2.1. Participants. Forty-four children ( 21 control ( 12 girls) and 23 with ADHD (7 girls); age range: 7-15 years old) composed the two groups of this cross-sectional study. Control participants (TD) were recruited through schools in the Montreal area. Participants with ADHD were recruited through a specialized clinic for ADHD in Montreal. All participants with ADHD had received a diagnosis by a specialized medical team based on DSM-IV-TR criteria (APA, 2000). In the following weeks, all parents filled out the Risk Factor Questionnaire (RFQ) [20] to confirm diagnosis in participants with ADHD as well as to exclude signs of the disorder in TD. Recruitment of patients was done over two and a half years, from 2009 to 2012. In addition, both sustained and selective attention were measured with the Continuous Performance Task (CPT) [21]. The ability to shift sets was measured by the Wisconsin Card Sorting Test (WCST) [22] and working memory with the Auditory Consonant Trigrams (ACT) test for children [23]. Mean composite IQ scores (K-BIT) [24] were also assessed in both groups. All adolescents were assessed in two sessions: neuropsychological and cerebral imagery while they were deprived of methylphenidate (MPH) for at least $24 \mathrm{hr}$. Children with motor, auditory or vision deficits, central nervous system abnormalities, and major health problems were excluded.

2.2. Functional Magnetic Resonance Imaging ( $f M R I$ ). The neural activation was assessed with fMRI. While in the scanner, participants were asked to judge whether two consecutive pictures told a congruent or incongruent story. There were 56 stories: 28 congruent (e.g., a picture of a girl grabbing a pint of milk followed by a picture of the same girl pouring milk in cereal: COherent condition) and 28 noncongruent (e.g., a picture of a girl grabbing a pint of milk followed by a picture of the same girl pouring water into a flower vase: INCOherent condition). The number of correct responses for each condition and the reaction time for each item were recorded. An fMRI blood oxygenation level dependent (BOLD) signal was recorded during performance of the task. A single-shot, gradient-recalled echo-planar imaging sequence (repetition time $=2000 \mathrm{~ms}$, echo time $=30 \mathrm{~ms}$, flip angle $=90$ degrees, and matrix $64 \times 64$ voxels) was used on a MRI Siemens TRIO system at 3.0 Tesla at Institut Universitaire Gériatrique de Montréal (IUGM). The images consisted of 32 continuous axial slices, with a $3 \mathrm{~mm} \times 3 \mathrm{~mm}$ in-plane resolution. The slice thickness was $3 \mathrm{~mm}$. During the run, 270 volumes were continuously acquired over a total duration of 540 seconds. A high-resolution T1-weighted scan $\left(1 \mathrm{~mm}^{3}\right.$ voxel size) was provided for each subject for anatomical coregistration. Prospective acquisition correction was applied to the images for better correction of head movement.

2.3. Data Analysis. Analyses of standardized neurological tests were done on the $T$-scores of all CPT and WCST measures and on raw scores for ACT. A $2 * 2$ ANOVA with the diagnosis and sex as between-subjects factors was performed on each measure. Interaction effects and pairwise comparisons were explored using the Bonferroni correction with the alpha level set to 0.05 . A log10 transformation was necessary to normalize the forethought data distribution and a $2 * 2$ ANCOVA with age as a covariate was performed for the number of correct responses (Hits), number of missed responses (Misses), reaction time (RT), and reaction time per correct responses (RT/Hit) for both the CO and INCO conditions. Previous to performing ANOVAs and ANCOVAs, assumptions of normality, homogeneity of variance, and presence of outliers were checked for each test and none were violated.

The fMRI data were processed using statistical parametric mapping (SPM8, Wellcome Department of Cognitive Neurology, London, UK). Individual cerebral activation of INCO $>\mathrm{CO}$ contrast served as the measure of forethought. A $2 \times 2$ ANCOVA was conducted in SPM8, with diagnosis and sex as independent factors and age as the covariate, to assess level of neural activation related to forethought. Because the literature is quite dispersed regarding the neural deficits in 
TABLE 1: Age and IQ of participants with ADHD and typically developing youth (TD).

\begin{tabular}{|c|c|c|c|c|c|c|c|c|}
\hline \multirow{2}{*}{$\mathrm{ADHD}$} & \multicolumn{4}{|c|}{ Boys } & \multicolumn{4}{|c|}{ Girls } \\
\hline & Mean & $\mathrm{SD}$ & Min & $\operatorname{Max}$ & Mean & $\mathrm{SD}$ & Min & Max \\
\hline Age & 10 & 2.3 & 7 & 15 & 11 & 1.51 & 7 & 14 \\
\hline K-Bit_Voc & 103 & 13.3 & 75 & 130 & 99 & 11.6 & 85 & 120 \\
\hline K-Bit_mat. & 110 & 16.3 & 91 & 150 & 107 & 12.8 & 93 & 127 \\
\hline K-Bit_total & 107 & 15.3 & 83 & 134 & 103 & 8 & 94 & 115 \\
\hline \multirow{2}{*}{ TD } & \multicolumn{4}{|c|}{ Boys } & \multicolumn{4}{|c|}{ Girls } \\
\hline & Mean & $\mathrm{SD}$ & Min & $\operatorname{Max}$ & Mean & $\mathrm{SD}$ & Min & Max \\
\hline Age & 11 & 1.2 & 9 & 12 & 11 & 1.9 & 7 & 14 \\
\hline K-Bit_Voc & 98 & 14.3 & 78 & 113 & 107 & 14.1 & 72 & 119 \\
\hline K-Bit_mat. & 108 & 16.3 & 69 & 136 & 118 & 12.2 & 96 & 136 \\
\hline K-Bit_total & 103 & 17.6 & 74 & 128 & 114 & 13.7 & 82 & 129 \\
\hline ANOVA & $F:$ Diagnosis & \multicolumn{2}{|c|}{$p$} & $F:$ Sex & $p$ & \multicolumn{2}{|c|}{$F$ : Group $\times$ Sex } & $p$ \\
\hline Age & 0.70 & \multicolumn{2}{|c|}{0.408} & 0.72 & 0.402 & \multicolumn{2}{|c|}{0.96} & 0.332 \\
\hline K-Bit_Voc & 0.21 & \multicolumn{2}{|c|}{0.651} & 0.31 & 0.541 & \multicolumn{2}{|c|}{2.40} & 0.129 \\
\hline K-Bit_mat. & 0.69 & \multicolumn{2}{|c|}{0.410} & 0.54 & 0.465 & \multicolumn{2}{|c|}{1.69} & 0.200 \\
\hline K-Bit_total & 0.54 & \multicolumn{2}{|c|}{0.466} & 0.61 & 0.439 & \multicolumn{2}{|c|}{2.60} & 0.114 \\
\hline
\end{tabular}

Note. K-Bit_Voc, Kaufman brief intelligence test_Vocabulary; K-Bit_mat., Kaufman brief intelligence test_Matrices; TD, typically developing youth; ADHD, youth with attention deficit and hyperactivity disorders.

All $p$ s are two-tailed.

ADHD (except for the frontostriatal network hypothesis), a whole brain analysis was conducted. The effects of diagnosis and sex were investigated in the contrasts [TD $>$ ADHD] and $[\mathrm{ADHD}>\mathrm{TD}$ ], and interaction effects in the following simple contrasts: [TD boys > ADHD boys], [TD girls > ADHD girls], [ADHD boys $>$ TD boys], and [ADHD girls $>$ TD girls]. Statistical tests were performed for group comparisons at a threshold level of $p=0.001$ uncorrected for multiple comparisons with a cluster threshold of 10 voxels. This more liberal threshold was used because the expected effects are broad but weak [25].

\section{Results}

3.1. Neuropsychological Profiles. Mean composite IQ scores (K-BIT) [24] varied between 103 and 114 and no IQ differences were found between diagnostic groups, sex or group-by-sex interaction (Table 1). Most neuropsychological measures indicated that boys with ADHD were in the mildly atypical clinical range pointing mostly to inattentive symptoms (CPT: omission, Hit RT, Hit RT SE). Their larger Tscores $(>60)$ on WCST also suggested lower mental flexibility (Table 2). Analysis of CPT indicated several main effects and sex-by-diagnostic interactions. A significant difference of omission was found between boys with ADHD and TD boys $(p=0.017)$ but not between TD girls and girls with ADHD. Regarding Hit RT, higher scores for boys with ADHD compared to girls with ADHD were observed $(p=0.01)$. For the Hit RT SE, the ADHD groups obtained higher scores and, specifically, boys with ADHD obtained significantly higher scores compared to TD boys $(p=0.016)$ and to TD girls $(p=0.037)$. The analysis of Hit RT Block change revealed that boys with ADHD obtained a higher score compared to girls with ADHD ( $p=0.024)$ but no difference appeared between girls and boys in the TD group. The same pattern of results occurred with Standard Errors for reaction time over Blocks (Hit SE Block change). Boys with ADHD displayed larger scores compared to girls with ADHD ( $p=0.004)$ and to TD girls $(p=0.003)$. A main effect of diagnostic was found for the Standard Error of reaction time slope over different interstimulus intervals (HIT SE ISI change); youth with ADHD demonstrated less consistency compared to TD groups $(p=0.006)$, a tendency more acute in boys with ADHD. The ACT analysis revealed a lesser performance for the groups with ADHD $(p=0.013)$ but no sex difference nor interaction between sex ${ }^{*}$ diagnostic group was observed. No subtest of WCST was statistically significant in terms of group differences (for brevity, we did not include these data in Table 2).

3.2. Forethought Performance. Behavioral performance results are presented in Table 3. No significant difference was found in the number of correct answers. However, reaction times (RT) analyses revealed group differences. For the $\mathrm{CO}$ condition, the TD group provided systematically faster responses (RT) $(p=0.003)$ and faster RT per hit $(p=0.008)$ compared to the group with ADHD. For the INCO condition, the TD group demonstrated marginally faster RT Inco/Hits compared to the ADHD group ( $p=0.073$ ). Although neither sex nor interaction effects were found, boys with ADHD showed systematically longer reaction times compared to girls with ADHD.

3.3. Neural Correlates of Forethought. Cerebral activation findings are presented in Table 4 . The diagnostic group contrast (TD > ADHD) showed significantly more activation 
TABLE 2: Neuropsychological profile of participants with ADHD and typically developing youth (TD).

\begin{tabular}{lcccccccccccc}
\hline & \multicolumn{2}{c}{ ADHD boys } & \multicolumn{2}{c}{ ADHD girls } & \multicolumn{2}{c}{ TD boys } & \multicolumn{2}{c}{ TD girls } & \multicolumn{2}{c}{$F$-value } \\
\hline ACT & Mean & SD & Mean & SD & Mean & SD & Mean & SD & $F:$ group & $F:$ sex & $F:(\mathrm{G}) \times(\mathrm{S})$ \\
Total & 32.13 & 11.2 & 44.50 & 8.4 & 48.44 & 8.3 & 48.50 & 15.9 & $5.35^{* *}$ & 2.00 & 1.86 \\
\hline CPT & & & & & & & & & & \\
$\quad$ Omission & 58.11 & 9.8 & 47.18 & 3.7 & 45.68 & 7.3 & 49.07 & 11.3 & 3.05 & 1.56 & $5.63^{*}$ \\
Commission & 53.54 & 5.4 & 56.78 & 6.3 & 46.58 & 21 & 59.96 & 18.5 & 0.16 & 3.10 & 1.16 \\
Hit RT & 58.42 & 12.3 & 40.42 & 5.2 & 48.89 & 6.9 & 48.21 & 13.5 & 0.06 & $6.65^{*}$ & $5.73^{*}$ \\
Hit RT SE & 61.46 & 10.5 & 49.42 & 11.1 & 47.10 & 7.8 & 49.56 & 12.1 & $4.21^{*}$ & 1.91 & $4.37^{*}$ \\
Hit RT ISI & 62.83 & 16.1 & 51.24 & 9.5 & 53.18 & 12.6 & 52.05 & 12.7 & 0.97 & 2.01 & 1.36 \\
Hit SE ISI & 59.48 & 11.7 & 55.24 & 11.5 & 45.09 & 9.5 & 49.67 & 8.8 & $8.51^{* *}$ & 0.00 & 1.69 \\
Hit RT Block & 60.45 & 11.3 & 48.2 & 13.4 & 50.07 & 13.6 & 48.77 & 11.9 & 0.47 & $5.96^{*}$ & $4.38^{*}$ \\
Hit SE Block & 59.08 & 7.9 & 40.95 & 11.3 & 48.88 & 11.7 & 49.11 & 7.53 & 0.12 & $9.46^{*}$ & $9.96^{*}$ \\
\hline
\end{tabular}

Note. ACT, Auditory Consonant Trigrams test; CPT, Continuous Performance Task; omission, number of times the subject fails to respond when target was presented; commission, number of response in absence of target; Hit RT, overall reaction time; Hit RT Block, slope of change in RTs over the six time blocks; Hit SE Block, slope of change in RTs standard errors over the six time blocks; Hit RT ISI, slope of change in RTs over the three interstimulus intervals; Hit SE ISI, slope of change in RTs SE over the three ISI; F: group corresponds to the Fisher test comparing youth with ADHD to youth without; $F$ : sex corresponds to the Fisher test comparing boys to girls and $F(\mathrm{G} \times \mathrm{S})$ corresponds to the Fisher measure observed for the interaction between the group and the sex. ${ }^{*} p<0.05 ;{ }^{* *} p<0.01$; all $p$ s are two-tailed.

TABLE 3: Forethought performance in participants with ADHD and typically developing (TD) youth.

\begin{tabular}{lccccccccccc}
\hline \multirow{2}{*}{ Measures } & \multicolumn{2}{c}{ ADHD boys } & \multicolumn{2}{c}{ ADHD girls } & \multicolumn{2}{c}{ TD boys } & \multicolumn{2}{c}{ TD girls } & \multicolumn{2}{c}{$2 \times 2$ ANCOVA } \\
& Mean & SD & Mean & SD & Mean & SD & Mean & SD & $F:(G)$ & $F:(S)$ & $F:(G) \times(S)$ \\
\hline Co_hit & 24.19 & 6.0 & 26.14 & 1.95 & 27.33 & 0.71 & 27 & 1.04 & 0.87 & 0.03 \\
Co_miss & 2.63 & 5.9 & 1.14 & 1.22 & 0.44 & 0.53 & 0.5 & 0.91 & 3.29 & 0.08 & 0.49 \\
RT_Co & 1412 & 256 & 1407 & 555 & 931.5 & 180 & 1150 & 270 & $10.02^{* *}$ & 2.49 & 0.21 \\
RT Co/hit & 74.94 & 69.8 & 55.15 & 26.7 & 34.07 & 6.48 & 42.73 & 10.3 & $7.93^{* *}$ & 0.87 & 1.39 \\
\hline Inco_hit & 23.38 & 6.5 & 26 & 1.9 & 27.22 & 0.8 & 26.75 & 1.2 & 0.74 & 0.07 & 0.53 \\
Inco_miss & 2.81 & 6.5 & 1.14 & 1.5 & 0.33 & 0.5 & 0.92 & 1.1 & 0.98 & 0.12 & 0.57 \\
RT_Inco & 1474 & 605 & 1341 & 551 & 996.6 & 228 & 1255 & 310 & 2.93 & 1.86 & 1.36 \\
RT Inco/hit & 95.9 & 134 & 52.49 & 24.6 & 36.59 & 8.14 & 47.22 & 12.9 & $3.38^{\dagger}$ & 0.40 & 1.88 \\
\hline
\end{tabular}

Note. Co_hit, good answer in authorized delay for coherent condition; Inco_hit, good answer in authorized delay for incoherent condition; Co_miss, error in authorized delay for coherent condition; Inco_miss, error in authorized delay for incoherent condition; RT_Co, mean reaction time (ms.) for coherent condition; RT Co/hit, mean reaction time (ms.) per hit for coherent condition; RT_Inco, mean reaction time (ms) for incoherent condition; RT Inco/hit, mean reaction (ms.) per hit for incoherent condition; F: group corresponds to the Fisher test comparing youth with ADHD to youth without; F: sex corresponds to the Fisher test comparing boys to girls and $F(\mathrm{G} \times \mathrm{S})$ corresponds to the Fisher measure observed for the interaction between the group and the sex.

${ }^{* *} p<0.01 ; \dagger=0.07$; all $p$ s are two-tailed.

in the right parietal postcentral gyrus (BA 1), left cingulate gyrus (BA 24), right middle frontal gyrus (MFG, BA 6), and the right culmen (cerebellum). No significant activation was observed in the opposite contrast (ADHD > TD). Relative to TD boys, boys with ADHD had extensive bilateral frontal and parietal hypoactivation, including bilateral superior frontal gyrus (BA 6), left inferior parietal lobule (IPL; BA 40), and right superior parietal lobule (SPL; BA 7). Boys with ADHD were also found to activate significantly more the right amygdala and left superior temporal gyrus than TD boys. Girls with ADHD demonstrated more scattered neural hypoactivation in comparison to TD girls including the right culmen, the right postcentral gyrus (BA 2), right middle temporal gyrus (MTG; BA 21), left claustrum (basal ganglia), and right inferior frontal gyrus (BA 47-10). There was no significant activation for the opposite contrast (ADHD girls $>$ TD girls).

\section{Discussion}

Our expectations that ADHD boys would demonstrate more deficits than ADHD girls in sustained and selective attention and in cerebral activation during forethought were partially supported by the present study. Indeed, ADHD boys expressed greater inattentiveness, greater impulsivity, and lesser vigilance than TD boys, while ADHD girls did not differ from TD girls. Boys with ADHD also showed greater variability. Overall, WM capacities were lower in ADHD relative to TD group, confirming previous reports $[3,10]$. Moreover, diagnosis by sex interactions observed on several occasions were compatible with studies reporting that boys with ADHD usually show more severe inattention symptoms [4] and greater executive deficits than girls with ADHD [15]. 
TABLE 4: Brain activations during forethought: contrast INCO-CO in participants with ADHD and typically developing (TD) youth.

\begin{tabular}{|c|c|c|c|c|c|c|}
\hline \multirow{2}{*}{ Comparison/region } & \multirow{2}{*}{$K$ (voxel) } & \multirow{2}{*}{$Z$ value } & \multicolumn{3}{|c|}{ MNI } & \multirow{2}{*}{$p$ value } \\
\hline & & & $x$ & $y$ & $z$ & \\
\hline \multicolumn{7}{|l|}{ TD-ADHD } \\
\hline Right parietal postcentral gyrus, BA 1 & 124 & 4.42 & 66 & -22 & 40 & 0.000 \\
\hline Left cingulate gyrus, BA 24 & 126 & 3.69 & -3 & -4 & 46 & 0.000 \\
\hline Right middle frontal gyrus, BA 6 & 92 & 4.10 & 27 & -7 & 67 & 0.000 \\
\hline Right anterior lobe, culmen & 64 & 3.62 & 30 & -52 & -32 & 0.000 \\
\hline \multicolumn{7}{|l|}{ ADHD-TD } \\
\hline \multicolumn{7}{|l|}{ No voxel activated } \\
\hline \multicolumn{7}{|l|}{ TD boys-ADHD boys } \\
\hline Bilateral superior frontal gyrus, BA 6 & 3300 & 4.36 & -18 & -4 & 58 & 0.000 \\
\hline Left inferior parietal lobule, BA 40 & 158 & 3.81 & -66 & -34 & 31 & 0.000 \\
\hline Right superior parietal lobule, BA 7 & 110 & 3.22 & 12 & -64 & 61 & 0.001 \\
\hline \multicolumn{7}{|l|}{ ADHD boys-TD boys } \\
\hline Right amygdala & 578 & 3.18 & 39 & -4 & -20 & 0.001 \\
\hline Left superior temporal gyrus & 114 & 3.15 & -39 & 8 & -23 & 0.001 \\
\hline \multicolumn{7}{|l|}{ TD girls-ADHD girls } \\
\hline Right cerebellum, culmen & 549 & 3.61 & 69 & -22 & 34 & 0.000 \\
\hline Right, postcentral gyrus, BA 2 & 180 & 3.25 & 30 & -55 & -29 & 0.001 \\
\hline Right middle temporal gyrus, BA 21 & 137 & 3.70 & 57 & -55 & -1 & 0.000 \\
\hline Left claustrum & 38 & 3.25 & -24 & 26 & -8 & 0.001 \\
\hline Right frontal gyrus, BA 10 & 32 & 3.15 & 45 & 53 & 1 & 0.001 \\
\hline Right, inferior frontal gyrus, BA 47 & 21 & 3.03 & 33 & 20 & -20 & 0.001 \\
\hline
\end{tabular}

ADHD girls-TD girls

No voxel activated

Note. Minimum cluster threshold = 10 voxels; TD, typically developing youth; ADHD, youth with attention deficit and hyperactivity disorders; INCO-CO, contrast between incoherent and coherent conditions; MNI, Montreal Neurological Institute; BA, Brodmann Area; $K$ (voxel) gives the number of voxels in each cluster; the $Z$ value is the $Z$-score given for that specific cluster with its $p$ value associated; $x, y, z$ corresponds to the $x, y, z$ MNI coordinates of the most central voxel of the cluster.

4.1. Forethought. The ADHD participants showed a tendency to be slower, but as accurate as the TD group on the forethought task. The forethought slowness was apparent especially in boys and was coherent with the CPT findings. As expected, extensive brain activation was observed in the TD group, including parietal and prefrontal regions as well as part of the cingulate gyrus and the cerebellum, during the forethought task performance. In contrast, the ADHD group showed no neural activation during forethought. This finding is consistent with reports of attenuated cerebral activation in ADHD tapping cognitive control [26, 27], working memory [2], and inhibition and vigilance/attention [28]. Overall, our study confirms the hypothesis of impairment in the frontalparietal pathway in ADHD [11, 12, 28]. Lateral portions of the PFC are part of the attentional network [29] and thus it is plausible that its malfunction is responsible for the encountered forethought slowness. Moreover, cerebral hypoactivation in ADHD youth is in accordance with their greater inattention and lack of vigilance, as well as weaker working memory.

4.2. Boys with ADHD. TD boys showed more extensive bilateral frontal activation relative to boys with ADHD, including the premotor cortex and the supplementary motor area
(SMA). Our results are compatible with findings observed in TD adolescent groups $[11,19,28]$ although no sex differentiation was made in these studies. Premotor cortex and SMA are involved in motor sequencing and planning [30], working memory [31], and visuospatial attention [32]. All these functions are essential for optimal performance of forethought. Moreover, hypoactivation of the SMA and the superior frontal gyrus has been accounted for motoric hyperactivity in ADHD [28].

TD boys also exhibited greater activation in the bilateral parietal cortex, left IPL and right SPL, relative to ADHD boys. The SPL is involved in visual representation of movements [33] and in WM related to motor sequence performance [34]. Thus, hypoactivation of SPL in boys with ADHD could have contributed to their impairment of forethought due to its link with WM. Similar results were observed by Dickstein et al. (2006) [11] where controls demonstrated significantly greater activation relative to ADHD in the left prefrontal and bilateral parietal lobes (IPL and SPL). The inferior part of SPL is related to inhibition, WM, and attention processes [28], all components of forethought. This region is part of the ventral attention network (VAN) that supports reorientation to behaviorally relevant external stimuli [35]. Thus, a deficient engagement of the VAN in ADHD may account 
for difficulties in adequately shifting attention to salient external stimuli [36]. Moreover, hypoactivation in VAN underpins ADHD-related deficits in detecting irregularities in the environment [28]. These considerations are relevant considering the present impairment in forethought, which involves a reflection on an incongruent succession of events.

Furthermore, boys with ADHD were found to hyperactivate a region adjacent to the medial temporal lobe, which is a part of the default mode network (DMN). This region was previously found to be hyperactivated in children with ADHD [28]. Fluctuations in activation of the DMN tend to correlate negatively with fluctuations in networks activated during task performance, typically the frontoparietal attention network [37]. The current findings in ADHD boys corroborate this pattern of activation. Indeed, their lesser functioning of the frontoparietal network might have impacted the DMN, resulting in disruption of the ongoing forethought performance. Together with the CPT results, this finding supports the recent perspective suggesting a link between variability and intrusion of "task-negative brain network" [38]. According to this perspective, RT variability is related to increased activity in DMN. The present study also supports the increasing recognition of motivational and emotional dysfunction in models of ADHD [39]. Boys with ADHD possibly perceived the task as more difficult or unpleasant, as it required a certain amount of time staying still and attentive, so that they had to rely on more activation of the amygdala to provide sufficient motivation.

4.3. Girls with ADHD. TD girls demonstrated widespread cerebral activation and there were no differences found for the ADHD girls > TD girls contrast. TD girls activated large portions of the cerebellum (culmen), a region activated in response to temporally unexpected stimuli [18]. There is mounting evidence that the basal ganglia and the cerebellum might project directly to one another [40,41]. Interestingly, both regions were activated in TD girls, but not in ADHD girls. Moreover, hypoactivation of the frontocerebellar network in girls with ADHD might have negatively impacted their ability to predict "when" events are going to occur and affect detection of violations of these predictions as suggested by previous studies [42, 43]. Because of its outputs to both the PFC and the basal ganglia, the cerebellum is in position to influence activity in circuits implicated in ADHD [42]. Moreover, because of its protracted development and sexual dimorphism, the cerebellum could play a crucial role in the neuropathology of girls with ADHD [44]. TD girls also activated the middle temporal gyrus (BA 21), a region activated in controls in the Durston et al. (2007) study [18], which investigated expectancy violation in ADHD, a condition similar to the incongruent situation of forethought.

TD girls also activated the claustrum, a region highlighted by Dickstein et al. (2006) [11] as being more activated in controls compared to participants with ADHD during EF performance. According to Crick and Koch (2005) [45], this part of basal ganglia plays a regulatory role in consciousness and cognition. Implication of the basal ganglia in conjunction with other regions might have helped TD girls in selecting the appropriate action and reject an incongruent action. Indeed the basal ganglia is believed to help in deciding among several possible behaviors at any given time $[46,47]$.

However, to fully establish the primacy of cerebral dysfunction in ADHD, future studies will need to employ a more comprehensive examination of executive function, using tasks known to produce consistent patterns of activity in other regions considered putative sources of dysfunction. Because the forethought task produced specific patterns of activity in boys and girls, our paradigm seems promising in further exploration of sex differences in the neural correlates of ADHD. Recent advances in brain imaging technology (e.g., diffusion tensor imaging and magnetoencephalography) allow us to examine more precisely and directly the relationships between brain regions during a cognitive task. A closer examination of the brain functional and anatomical connectivity would help in identifying entire neural networks underlying cognitive deficits in ADHD. Future research, including girls in the field of ADHD, promises exciting results linking the genomic, structural, and functional changes in the brain of individuals with ADHD. Advances in understanding of the ADHD neurobiology will hopefully help in developing new psychotherapeutic interventions and in identifying more targeted pharmacotherapies so that child psychiatrists can better manage their patients.

In conclusion, the present findings point to greater $\mathrm{EF}$ deficits in boys compared to girls with ADHD. Moreover, boys with ADHD exhibited cerebral hypoactivation in the frontoparietal network. On the other hand, they hyperactivated cerebral regions linked to motivation. Taken together, these findings indicate that boys with ADHD show decreased attention processing that seems compensated by the use of supplemental motivation to perform as accurately as their TD counterparts. However, this compensation comes with a time cost. Girls with ADHD displayed more dispersed decreased neural activation in the frontocerebellar and frontoparietal systems compared to the TD girls. This suggests that they rely on different circuits to make decisions about incongruent situations with a related time cost. Overall, the present study supports the hypothesis of different neuropsychological and neurofunctional profiles in boys and girls with ADHD. This brings to light the importance of considering both genders while investigating ADHD in order to better understand the disorder and propose clinical intervention more specific to the gender.

\section{Competing Interests}

The authors declare that they have no competing interests.

\section{References}

[1] American Psychiatric Association, Diagnostic and Statistical Manual of Mental Disorders, American Psychiatric, Arlington, Va, USA, 2013.

[2] E. M. Valera, S. V. Faraone, K. E. Murray, and L. J. Seidman, "Meta-analysis of structural imaging findings in attentiondeficit/hyperactivity disorder," Biological Psychiatry, vol. 61, no. 12, pp. 1361-1369, 2007. 
[3] R. A. Barkley, Attention-Deficit Hyperactivity Disorder: A Handbook for Diagnosis and Treatment, Guilford, New York, NY, USA, 3rd edition, 2005.

[4] J. Gershon, "A meta-analytic review of gender differences in ADHD," Journal of Attention Disorders, vol. 5, no. 3, pp. 143-154, 2002.

[5] E. W. Skogli, M. H. Teicher, P. N. Andersen, K. T. Hovik, and M. Øie, "ADHD in girls and boys-gender differences in co-existing symptoms and executive function measures," $B M C$ Psychiatry, vol. 13, no. 1, article 298, 2013.

[6] J. J. Rucklidge, "Gender differences in attentiondeficit/hyperactivity disorder," Psychiatric Clinics of North America, vol. 33, no. 2, pp. 357-373, 2010.

[7] S. L. Andersen and M. H. Teicher, "Sex differences in dopamine receptors and their relevance to ADHD," Neuroscience \& Biobehavioral Reviews, vol. 24, no. 1, pp. 137-141, 2000.

[8] S. Hosenbocus and R. Chahal, "A review of executive function deficits and pharmacological management in children and adolescents," Journal of the Canadian Academy of Child and Adolescent Psychiatry, vol. 21, no. 3, pp. 223-229, 2012.

[9] J. E. Schreiber, K. L. Possin, J. M. Girard, and C. ReyCasserly, "Executive function in children with attention deficit/hyperactivity disorder: the NIH EXAMINER battery," Journal of the International Neuropsychological Society, vol. 20, no. 1, pp. 41-51, 2014.

[10] R. Martinussen, J. Hayden, S. Hogg-Johnson, and R. Tannock, "A meta-analysis of working memory impairments in children with attention-deficit/hyperactivity disorder," Journal of the American Academy of Child \& Adolescent Psychiatry, vol. 44, no. 4, pp. 377-384, 2005.

[11] S. G. Dickstein, K. Bannon, F. Xavier Castellanos, and M. P. Milham, "The neural correlates of attention deficit hyperactivity disorder: an ALE meta-analysis," Journal of Child Psychology and Psychiatry and Allied Disciplines, vol. 47, no. 10, pp. 10511062, 2006.

[12] K. Rubia, A. B. Smith, R. Halari et al., "Disorder-specific dissociation of orbitofrontal dysfunction in boys with pure conduct disorder during reward and ventrolateral prefrontal dysfunction in boys with pure ADHD during sustained attention," The American Journal of Psychiatry, vol. 166, no. 1, pp. 8394, 2009.

[13] U. P. Ramtekkar, A. M. Reiersen, A. A. Todorov, and R. D. Todd, "Sex and age differences in attention-deficit/hyperactivity disorder symptoms and diagnoses: implications for DSM-V and ICD-11," Journal of the American Academy of Child and Adolescent Psychiatry, vol. 49, no. 3, pp. 217.e3-228.e3, 2010.

[14] E. M. Valera, A. Brown, J. Biederman et al., "Sex differences in the functional neuroanatomy of working memory in adults with ADHD," The American Journal of Psychiatry, vol. 167, no. 1, pp. 86-94, 2010.

[15] M. A. Sheridan, S. Hinshaw, and M. D’Esposito, "Efficiency of the prefrontal cortex during working memory in attentiondeficit/hyperactivity disorder," Journal of the American Academy of Child \& Adolescent Psychiatry, vol. 46, no. 10, pp. 1357-1366, 2007.

[16] F. E. Dupuy, R. J. Barry, A. R. Clarke, R. McCarthy, and M. Selikowitz, "Sex differences between the combined and inattentive types of attention-deficit/hyperactivity disorder: an EEG perspective," International Journal of Psychophysiology, vol. 89, no. 3, pp. 320-327, 2013.
[17] R. A. Barkley, "Attention-deficit/hyperactivity disorder, selfregulation, and executive functioning," in Handbook of SelfRegulation: Research, Theory, and Applications, K. D. Vohs and F. B. Roy, Eds., pp. 551-564, Guilford, New York, NY, USA, 2011.

[18] S. Durston, M. C. Davidson, M. J. Mulder et al., "Neural and behavioral correlates of expectancy violations in attentiondeficit hyperactivity disorder," Journal of Child Psychology and Psychiatry and Allied Disciplines, vol. 48, no. 9, pp. 881-889, 2007.

[19] H. Poissant, A. Mendrek, and N. Senhadji, "Neural correlates of forethought in ADHD," Journal of Attention Disorders, vol. 18, no. 3, pp. 258-267, 2014.

[20] H. Poissant and S. Lecomte, "Risk factors in families of children with attention deficit/hyperactivity disorders: Quebec data," Journal of the Canadian Academy of Child and Adolescent Psychiatry, vol. 16, no. 1, pp. 9-17, 2007.

[21] C. K. Conners, J. N. Epstein, A. Angold, and J. Klaric, "Continuous performance test performance in a normative epidemiological sample," Journal of Abnormal Child Psychology, vol. 31, no. 5, pp. 555-562, 2003.

[22] R. K. Heaton, W. Miller, M. J. Taylor, and I. Grant, Wisconsin Card Sorting Test, PAR Staff, 2004.

[23] C. Paniak, H. B. Miller, D. Murphy, A. Andrews, and J. Flynn, "Consonant trigrams test for children: development and norms," Clinical Neuropsychologist, vol. 11, no. 2, pp. 198-200, 1997.

[24] A. S. Kaufman and N. L. Kaufman, Kaufman Brief Intelligence Test, John Wiley \& Sons, New York, NY, USA, 2004.

[25] R. C. Wolf, M. M. Plichta, F. Sambataro et al., "Regional brain activation changes and abnormal functional connectivity of the ventrolateral prefrontal cortex during working memory processing in adults with attention-deficit/hyperactivity disorder," Human Brain Mapping, vol. 30, no. 7, pp. 2252-2266, 2009.

[26] K. P. Schulz, J. Fan, C. Y. Tang et al., "Response inhibition in adolescents diagnosed with attention deficit hyperactivity disorder during childhood: an event-related fMRI study," The American Journal of Psychiatry, vol. 161, no. 9, pp. 1650-1657, 2004.

[27] S. J. Suskauer, D. J. Simmonds, S. Fotedar et al., "Functional magnetic resonance imaging evidence for abnormalities in response selection in attention deficit hyperactivity disorder: differences in activation associated with response inhibition but not habitual motor response," Journal of Cognitive Neuroscience, vol. 20, no. 3, pp. 478-493, 2008.

[28] S. Cortese, C. Kelly, C. Chabernaud et al., "Toward systems neuroscience of ADHD: a meta-analysis of 55 fMRI studies," The American Journal of Psychiatry, vol. 169, no. 10, pp. 10381055, 2012.

[29] J. M. Shine, G. M. Halliday, S. L. Naismith, and S. J. G. Lewis, "Visual misperceptions and hallucinations in Parkinson's disease: dysfunction of attentional control networks?" Movement Disorders, vol. 26, no. 12, pp. 2154-2159, 2011.

[30] A. Bischoff-Grethe, K. M. Goedert, D. T. Willingham, and S. T. Grafton, "Neural substrates of response-based sequence learning using fMRI," Journal of Cognitive Neuroscience, vol. 16, no. 1, pp. 127-138, 2004.

[31] C. Ranganath, M. K. Johnson, and M. D’Esposito, "Prefrontal activity associated with working memory and episodic longterm memory," Neuropsychologia, vol. 41, no. 3, pp. 378-389, 2003.

[32] A. C. Nobre, G. N. Sebestyen, D. R. Gitelman, M. M. Mesulam, R. S. J. Frackowiak, and C. D. Frith, "Functional localization of 
the system for visuospatial attention using positron emission tomography," Brain, vol. 120, no. 3, pp. 515-533, 1997.

[33] A. Solodkin, P. Hlustik, E. E. Chen, and S. L. Small, "Fine modulation in network activation during motor execution and motor imagery," Cerebral Cortex, vol. 14, no. 11, pp. 1246-1255, 2004.

[34] M. J. Catalan, M. Honda, R. A. Weeks, L. G. Cohen, and M. Hallett, "The functional neuroanatomy of simple and complex sequential finger movements: a PET study," Brain, vol. 121, no. 2, pp. 253-264, 1998.

[35] M. Corbetta, G. Patel, and G. L. Shulman, "The reorienting system of the human brain: from environment to theory of mind," Neuron, vol. 58, no. 3, pp. 306-324, 2008.

[36] P. Helenius, M. Laasonen, L. Hokkanen, R. Paetau, and M. Niemivirta, "Neural correlates of late positivities associated with infrequent visual events and response errors," NeuroImage, vol. 53, no. 2, pp. 619-628, 2010.

[37] E. J. S. Sonuga-Barke, "Editorial: ADHD as a reinforcement disorder-moving from general effects to identifying (six) specific models to test," Journal of Child Psychology and Psychiatry, vol. 52, no. 9, pp. 917-918, 2011.

[38] L. Tamm, M. E. Narad, T. N. Antonini, K. M. O’Brien, L. W. Hawk Jr., and J. N. Epstein, "Reaction time variability in ADHD: a review," Neurotherapeutics, vol. 9, no. 3, pp. 500-508, 2012.

[39] E. J. S. Sonuga-Barke and F. X. Castellanos, "Spontaneous attentional fluctuations in impaired states and pathological conditions: a neurobiological hypothesis," Neuroscience \& Biobehavioral Reviews, vol. 31, no. 7, pp. 977-986, 2007.

[40] A. C. Bostan, R. P. Dum, and P. L. Strick, "The basal ganglia communicate with the cerebellum," Proceedings of the National Academy of Sciences of the United States of America, vol. 107, no. 18, pp. 8452-8456, 2010.

[41] E. Hoshi, L. Tremblay, J. Féger, P. L. Carras, and P. L. Strick, "The cerebellum communicates with the basal ganglia," Nature Neuroscience, vol. 8, no. 11, pp. 1491-1493, 2005.

[42] S. Durston, J. van Belle, and P. de Zeeuw, "Differentiating frontostriatal and fronto-cerebellar circuits in attentiondeficit/hyperactivity disorder," Biological Psychiatry, vol. 69, no. 12, pp. 1178-1184, 2011.

[43] R. B. Ivry and R. M. C. Spencer, "The neural representation of time," Current Opinion in Neurobiology, vol. 14, no. 2, pp. 225232, 2004.

[44] H. Tiemeier, R. K. Lenroot, D. K. Greenstein, L. Tran, R. Pierson, and J. N. Giedd, "Cerebellum development during childhood and adolescence: a longitudinal morphometric MRI study," NeuroImage, vol. 49, no. 1, pp. 63-70, 2010.

[45] F. C. Crick and C. Koch, "What is the function of the claustrum?" Philosophical Transactions of the Royal Society B: Biological Sciences, vol. 360, no. 1458, pp. 1271-1279, 2005.

[46] A. Stocco, C. Lebiere, and J. R. Anderson, "Conditional routing of information to the cortex: a model of the basal Ganglia's role in cognitive coordination," Psychological Review, vol. 117, no. 2, pp. 541-574, 2010.

[47] V. S. Chakravarthy, D. Joseph, and R. S. Bapi, "What do the basal ganglia do? A modeling perspective," Biological Cybernetics, vol. 103, no. 3, pp. 237-253, 2010. 


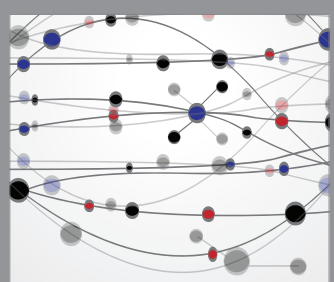

The Scientific World Journal
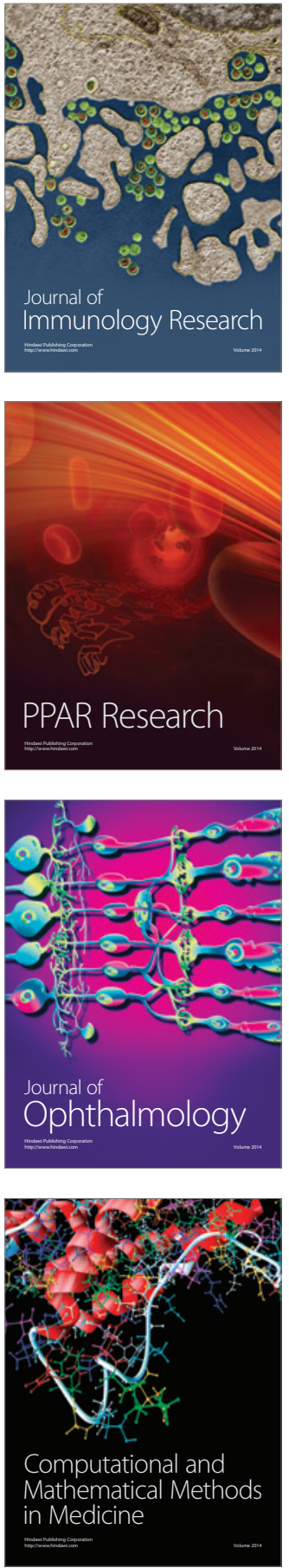

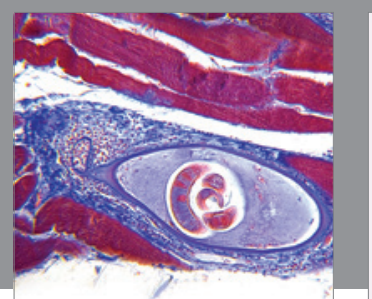

Gastroenterology Research and Practice

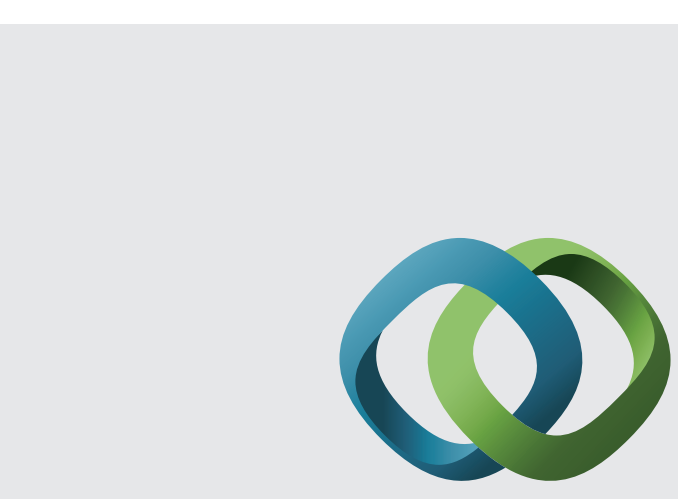

\section{Hindawi}

Submit your manuscripts at

http://www.hindawi.com
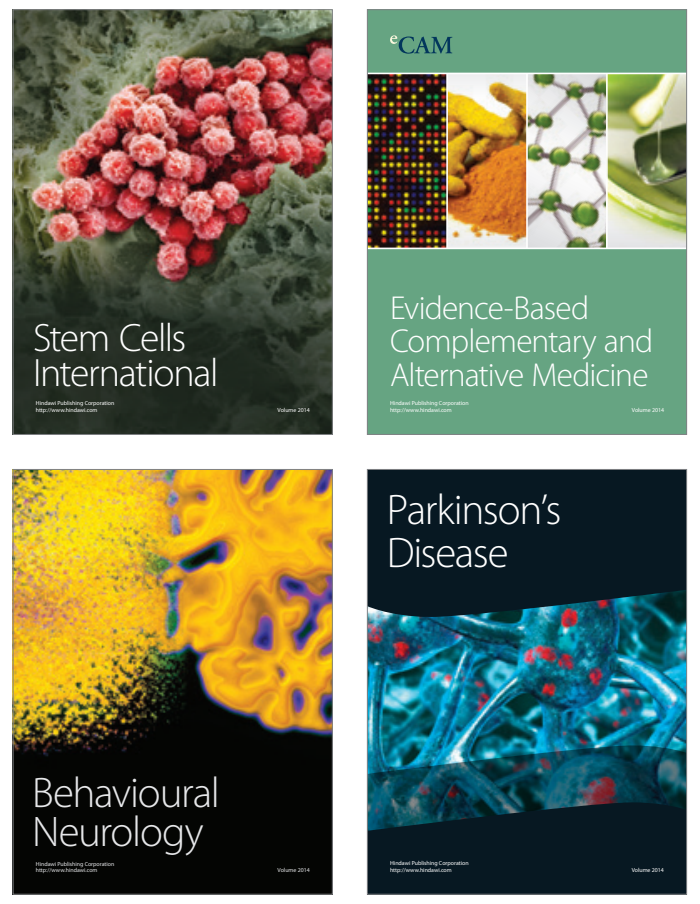
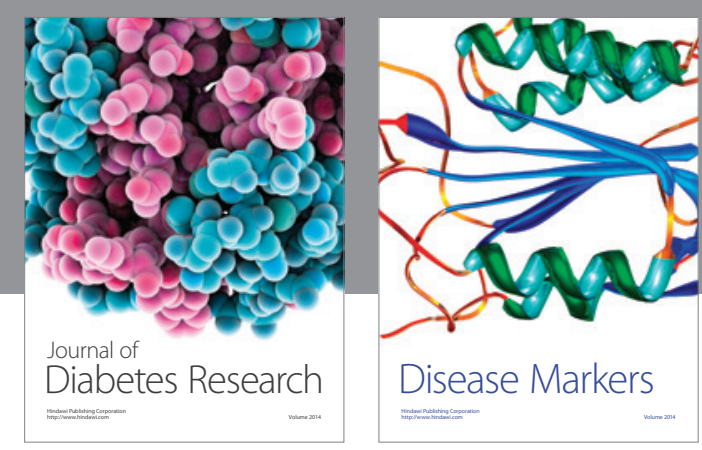

Disease Markers
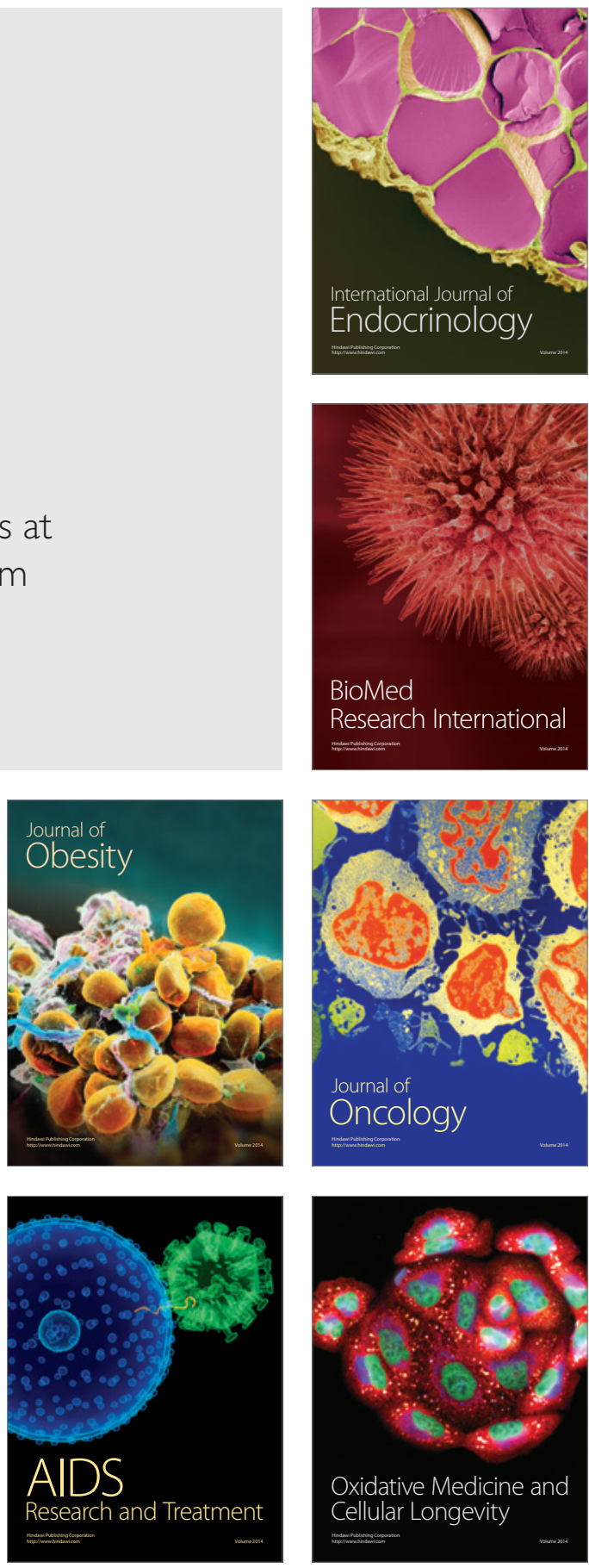